\title{
Face processing: Human perception and principal components analysis
}

\author{
PETER J. B. HANCOCK \\ University of Stirling, Stirling, Scotland \\ A. MIKE BURTON \\ University of Glasgow, Glasgow, Scotland \\ and \\ VICKI BRUCE \\ University of Stirling, Stirling, Scotland
}

\begin{abstract}
Principal components analysis (PCA) of face images is here related to subjects' performance on the same images. In two experiments subjects were shown a set of faces and asked to rate them for distinctiveness. They were subsequently shown a superset of faces and asked to identify those that had appeared originally. Replicating previous work, we found that hits and false positives (FPs) did not correlate: Those faces easy to identify as being "seen" were unrelated to those faces easy to reject as being "unseen." PCA was performed on three data sets: (1) face images with eye position standardized, (2) face images morphed to a standard template to remove shape information, and (3) the shape information from faces only. Analyses based on PCA of shape-free faces gave high predictions of FPs, whereas shape information itself contributed only to hits. Furthermore, whereas FPs were generally predictable from components early in the PCA, hits appeared to be accounted for by later components. We conclude that shape and "texture" (the image-based information remaining after morphing) may be used separately by the human face processing system, and that PCA of images offers a useful tool for understanding this system.
\end{abstract}

Psychological research on face recognition has tended to divide into two broad approaches. One approach has been to concentrate on cognitive processes following perception and to develop information processing models (see, e.g., Bruce \& Young, 1986; Burton, Bruce, \& Johnston, 1990; Ellis, 1986; Hay \& Young, 1982; Young \& Bruce, 1991). This approach has been very successful in delineating the stages involved in face recognition; however, each of these models has assumed some perceptual processing prior to input. Indeed, some information processing models explicitly require input in the form of componential face primitives, but remain uncommitted about the nature of these primitives (see, e.g., Burton, 1994; Farah, O'Reilly, \& Vecera, 1993; Valentine, 1991).

Other research by psychologists has investigated the perceptual processing of face patterns, demonstrating, for example, how faces seem to be analyzed holistically rather than by being decomposed into discrete local features (see, e.g., Bartlett \& Searcy, 1993; Rhodes, Brake, \& Atkinson, 1993; Tanaka \& Farah, 1993; Young, Hellawell,

We are grateful to I. Craw and N. Costen for providing the images used in these experiments and to D. Carson, who ran Experiments 1 and 2. The manuscript was improved following comments from G. Loftus, M. Reinitz, and P. Dixon. This research was supported by an SERC grant to A.M.B., V.B., and I. Craw (No. GRH 93828). Correspondence should be addressed to P. J. B. Hancock, Department of Psychology, University of Stirling, Stirling, Scotland, FK9 4LA, UK (e-mail: pjh@psych.stir.ac.uk)
\& Hay, 1987). However, this research tends not to consider the way in which such perceptual processes deliver codes suitable for the task of recognizing individual faces. In contrast, a growing body of research by computer scientists and engineers has addressed this question explicitly in the quest for artificial face recognition systems suitable for security and forensic applications. This research has progressed largely without considering the psychological plausibility of the coding schemes employed.

The aim of the work presented in this paper is to examine the psychological plausibility of one such scheme for coding face images for recognition--the principal components analysis (PCA) of face images (Kirby \& Sirovich, 1990; Turk \& Pentland, 1991). PCA has a number of characteristics that make it attractive as a potential model for human face image coding, as we elaborate below, and recent work (e.g., O'Toole, Deffenbacher, Valentin, \& Abdi; 1994) has shown that PCA of a set of face images does a good job of accounting for some aspects of human memory performance with these same images. Our work with PCA builds on these earlier studies and shows that it is possible to improve the psychological predictive power of a PCA-based model by incorporating a preprocessing stage in which the spatial deviation of each face shape from the average (its "shape") is coded separately.

In this introduction we will first consider the evidence that PCA belongs to the right class of image analysis 
schemes for psychological plausibility and then consider details of the approach itself. We will then consider the way in which a PCA-based system might in principle allow us to implement psychological theories of face space and norm-based coding. This introduction motivates the new experimental and image analysis work presented in this paper.

\section{Evidence for Image-Based Face Coding Schemes}

Recently a number of researchers have attempted to understand how the human visual system analyzes and stores face images in order to relate image analysis to psychological aspects of face processing. In these studies, researchers have evaluated a particular candidate for face primitives with respect to human performance data on faces. For example, a number of researchers have examined the potential of simple Euclidean measures such as length of nose, width of mouth, and so on (see, e.g., Rhodes, 1988). Combinations of such measures taken from a large corpus of faces have been used to derive indices corresponding to human judgments of sex (Bruce, Burton, et al., 1993; Burton, Bruce, \& Dench, 1993) and to human judgments of distinctiveness (Bruce, Burton, $\&$ Dench, 1994). In both these projects, the authors concluded that primitives based on these Euclidean distances alone are probably insufficient for understanding internal representations of faces.

A rather different approach, influenced by the 3-D model-based approach to visual object recognition (see, e.g., Biederman, 1987) was taken by Bruce, Coombes, and Richards (1993). These authors examined the psychological plausibility of a 3-D surface-based coding scheme in which each face was described as a spatial distribution of 3-D surface primitives such as peaks, pits, valleys, and ridges. While it can be shown that variations in surface descriptions covary with psychological dimensions (Bruce, Burton, et al., 1993; Bruce, Coombes, \& Richards, 1993), the observation that face recognition is highly error prone when faces are displayed as surface images devoid of texture or pigmentation (Bruce et al., 1991) suggests that the face primitives used for recognition of faces cannot be based on 3-D shape descriptions alone.

For a coding scheme to have psychological plausibility, it must be able to account for the difficulty that people have with recognizing faces shown in certain formats. For example, recognition is extremely difficult when faces are portrayed as line drawings in which major (e.g., mouth) and minor (e.g., wrinkles) face features are traced (Bruce, Hanna, Dench, Healy, \& Burton, 1992;
Davies, Ellis, \& Shepherd, 1978). It is difficult to explain why such drawings are so difficult to recognize if our coding of faces is based on Euclidean metric measurements, which should be preserved in such drawings. Recognition of line drawings of faces improves dramatically if they contain information about areas of relative dark and light from the original image, as well as edges (Bruce et al., 1992). Similarly, face recognition is dramatically impaired if faces are shown in photographic negatives (Bruce, Burton, et al., 1993; Hayes, Morrone, \& Burr, 1986), even though a negative image of a face preserves the spatial layout of the face. Such observations suggest that human facial image coding incorporates information about image intensities themselves, and not just the spatial layout of changes in image intensity. The relative pattern of light and dark within a face conveys important discriminating information about such things as hair and skin color, and 3-D shape from patterns of shading and shadows.

PCA is one example of a scheme that codes image intensities and that does not decompose faces into localized features. O'Toole and her colleagues have performed PCA on facial images and related these to human performance on recognition of own and other-race facial images (O'Toole et al., 1994), and on human performance in sex judgments (Abdi, Valentin, Edelman, \& O'Toole, 1995). Results from these studies have been promising; it appears that PCA may provide a plausible candidate for the notion of facial primitives.

\section{The PCA Approach and Shape-Free PCA}

The basic technique of PCA on images of faces is now well developed (Kirby \& Sirovich, 1990; Turk \& Pentland, 1991). A set of facial images is collected and registered (e.g., by normalizing the position of the eyes for each face). These images may then be considered as a one-dimensional array of pixel values (gray levels). Correlations are taken between these images, and the coefficients of the principal components (eigenvectors, sometimes known as eigenfaces) are extracted. The coefficients have the same dimension as each of the input images, and may be displayed (Figure 1). As the images are preprocessed to have a zero mean, the eigenfaces code deviations from the mean, and have a rather ghostly appearance. The first eigenface codes the direction of maximum variance in these images. The second codes the direction of maximum variance after that accounted for by the first has been removed. They are difficult to interpret visually, but some features may be observed. In the forehead area of the images in Figure 1, the first and third com-
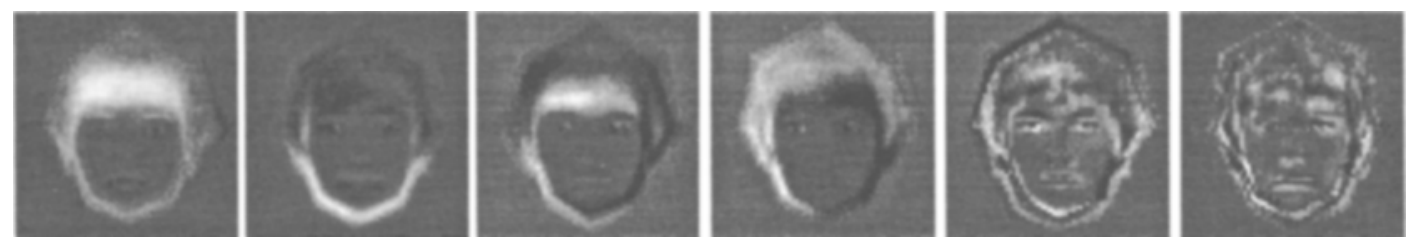

Figure 1. The first 4 , the 20 th, and the 40 th eigenfaces generated from the complete set of 174 full images. 
ponents appear to reflect overall fringe length, whereas the second and fourth are lopsided, corresponding to individuals with hair on only one side of their forehead. Note that the sign with which the images are displayed is not significant, so we cannot say that the first component makes the whole forehead lighter, while the second makes the left (as we look at it) side darker, but only that the first is symmetrical, the second not. Note also that the later eigenfaces apparently carry finer detail. This is consistent with the suggestion by O'Toole et al. (1994) that the later components carry information about identity.

Each of the faces in the corpus generating the components may then be reconstructed by a weighted sum of the eigenvectors. Similarly, new faces may be stored as a weighted sum of eigenfaces. This offers a mechanism for compact storage of face images. A full PCA of a set of 100 faces will generate 99 components (plus the mean pixel values). Because the early components capture most of the variance, it may be possible to produce visually acceptable regenerations of the images from, say, only 50 components. This almost halves the required storage, requiring only the 50 eigenface images, and then 50 component values for each face. New faces may then be coded using the existing 50 eigenfaces, requiring storage of only the 50 component values for each additional face. How well a new face is regenerated will depend on its match to the original corpus. A face that differs significantly, for instance, in race, may well be rather poorly coded. This characteristic of PCA will be exploited in the studies reported here.

The compact principal component coding of a face forms the basis of PCA-based face recognition. The match between a novel face image and an existing database is performed in the reduced space of the coded images. Our interest here is not an attempt at face recognition per se, but an investigation of the psychological plausibility of eigenface-based representation of faces. Some researchers have already noted that particular eigenfaces appear to code particular facial characteristics. For example, O'Toole, Abdi, Deffenbacher, and Bartlett (1991) have shown that information about the sex of a face may be present in the early eigenfaces (those with the largest eigenvalues).

Although analysis based on image characteristics may have psychological plausibility, it brings with it a number of problems that arise from the specific characteristics of the images used to create the corpus of faces. It is usually acknowledged that some preprocessing of images is required, for example, in order to normalize intensity values. In the following experiments, we present data from a different type of preprocessing. Before subjecting face images to PCA, we first eliminate any deviation they have from the average shape of the corpus. This technique, introduced by Craw and Cameron (1991), is illustrated in Figure 2. One first defines a set of key points that are located for each face. An average value is calculated for each of these points, and a grid is constructed, corresponding to the average face shape. The same points are used to construct a grid for each facial image. This grid is then morphed into the averaged shape using simple interpolation. The result is an image that has standard shape, called a "shape-free face" by Craw and Cameron.

There are several computational advantages of using shape-free faces for PCA. Previous researchers have sub-
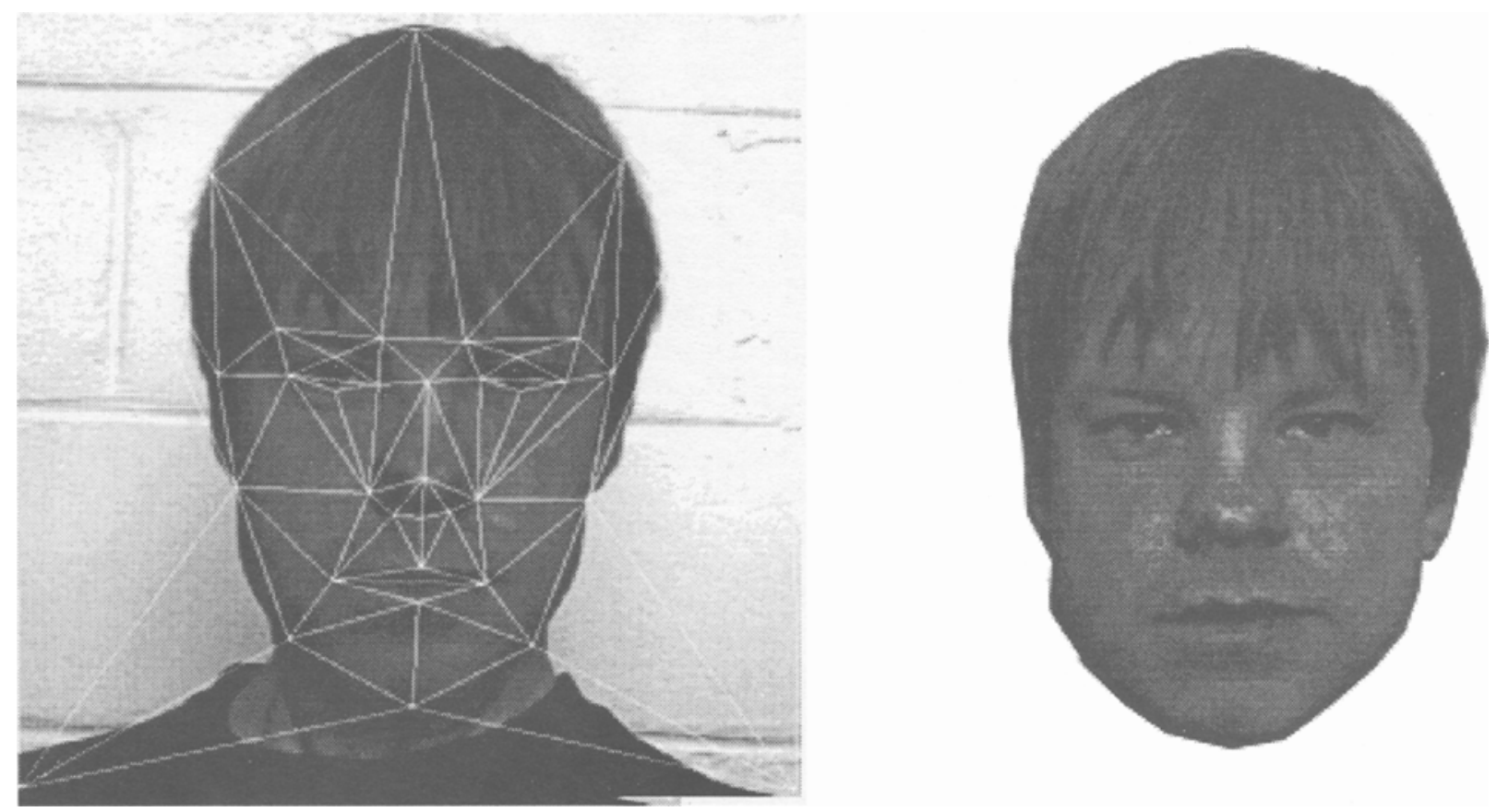

Figure 2. (left) The set of control points used to define the positions of features around each face. (right) The same face, morphed to the average shape, with background removed. 
jected whole images to principal components analysis, including backgrounds that are sometimes noisy (e.g., Turk \& Pentland, 1991). Once all faces have the same shape, it is simple to separate face from background and to analyze only the face. Second, when reconstructing a face, one can add the components derived from shapefree faces in linear fashion without changing the overall shape of the face. Combination of components from shaped images leads to blurred edges due to contributions from components derived over a range of face shapes. Finally, in extracting the shape from a face, one can independently examine the contributions of shape-the spatial deviation of each face from the average - and "texture." We use the word texture in this paper as a shorthand way to cover all the image information that remains in the shape-free face-that is, color information and the fine-scale features unaffected by the shape averaging. It is important to note that our use of the word texture is more restricted than its more conventional usage in psychology. As we will elaborate next, the use of PCA to derive a set of dimensions along which faces vary, and the separate analysis of shape and texture, provide a possible means of implementing psychological theories of face space and norm-based coding using a coding scheme that is sensitive to low-level image properties.

\section{Face Space and Norm-Based Coding}

Much of the recent literature on face recognition has (often implicitly) relied on the notion of face spacethat is, the notion that there are a number of dimensions along which faces vary, and that a face can be uniquely represented as a point, or vector, in that space. This notion was made explicit by Valentine (1991), who provided an account of facial distinctiveness in these terms (among other effects). The phenomena associated with distinctiveness in face recognition are well documented. Unfamiliar faces that subjects have rated as being distinctive tend to be remembered better (in a recognition paradigm) than faces that have been rated as typical. Familiar faces that have been rated as distinctive tend to be recognized (as familiar) faster than familiar faces that have been rated as typical (Valentine \& Bruce, 1986b). The explanation of these effects in terms of face space proceeds as follows. Faces rated as typical will tend to have common values on dimensions defining face space. This means that typical faces will be clustered together. Distinctive faces, on the other hand, will tend to be relatively isolated in face space: a corpus of distinctive faces, by definition, has few faces that look similar. Valentine (1991) has argued that the relative isolation of distinctive faces makes them easier to recognize than typical faces, because there will be fewer competitor faces in the relevant region of face space.

This discussion of face space has proceeded without any reference to the actual nature of the dimensions along which faces vary: What are the dimensions of this space? The central aim of this paper is to examine the possibility that the dimensions along which faces vary can be captured in a PCA of images. This is a very dif- ferent approach from that taken by other workers in this field. For example, discussing the nature of face space, Valentine (1991) stated that "previous work using multidimensional scaling techniques suggests that the principal dimensions needed would represent hair colour and length, face shape and age" (p. 166). In this paper, we examine the possibility that faces may be coded on dimensions extracted from PCA of face images, rather than corresponding to these commonsense dimensions. Of course, this does not rule out the possibility that dimensions extracted from PCA may themselves have a strong relation with dimensions such as hair length, used in everyday life descriptions of faces.

Among adherents to this view of face space, there is disagreement about the nature of representation. Valentine (1991) has contrasted norm-based and exemplarbased coding. Norm-based coding refers to the idea that faces are encoded as a vector in face space, with reference to a central norm calculated as the average of the known population of faces. This idea has been used to account for caricature effects in face recognition. Rhodes, Brennen, and Carey (1987) showed that faces distorted away from a central mean may, in some circumstances, be recognized more accurately (and faster) than a veridical image of a face (see also Benson \& Perrett, 1991). This has led to the suggestion that faces are coded as $d e-$ viations from a central tendency of one's known population of faces. In contrast to norm-based coding, some researchers have used the idea of exemplar-based coding (Nosofsky, 1986) to capture the notion that faces are coded in face space, but without reference to a central norm. Valentine and Endo (1992) have produced some preliminary evidence, based on the other-race effect, that faces may be coded without reference to a central norm.

In the absence of any concrete proposals about the nature of underlying dimensions of face space, it is difficult to separate predictions from the norm-based and exemplar-based views. In this paper we will not address this distinction. However, it appears to us that the use of PCA on shape-free faces does, for the first time, offer the possibility of a computational account of norm-based coding. If there is, indeed, a central norm for our set of known faces, one way in which faces deviate from this norm consists in the differences among their shapes. In the analyses presented in the second half of this paper we shall show that it is possible to examine this type of deviation independently from deviation due to the texture of a face (e.g., the variance due to different coloration). By preprocessing facial images to remove variance in shape (and by separately analyzing this information), it is possible to measure variability from a central norm due to these different types of information.

In this paper we test the ability of a PCA-based coding scheme operating on whole faces and separately on "shape" and "shape-free" faces to account for variations in the rated distinctiveness of face images and to account for variations in human memory performance with the same face images. Before describing these investigations, however, we must introduce some complications that 
arise in the relationship between rated distinctiveness and measures of memory.

\section{Distinctiveness}

It is most people's intuition that there should be a straightforward mapping between the dimension that subjects respond to when asked to rate face typicality/ distinctiveness, and memory performance with faces. Faces that are highly distinctive in appearance (e.g., a face with a long red beard) should —one might thinkbe highly memorable and rarely give rise to false alarms when presented as distractors. Faces that are very typical in appearance should be less memorable but more likely to give rise to false alarms. In fact, this intuition turns out to be wrong. Vokey and Read (1992) discovered that rated typicality (cf. distinctiveness) is in fact composed of two orthogonal components, one coding familiarity, and another coding memorability. By decomposing subjects' ratings of faces, they showed that the tendency to rate a face as familiar ("context-free familiarity") dissociates from the tendency to rate the face as being memorable. This finding was replicated by O'Toole et al. (1994). These researchers found that for faces from one's own race, ratings to the question, "Is the face confusable with someone you know?" dissociate from ratings to the question, "Is the face easy to remember?" Bruce et al. (1994) revealed a similar disssociation in memory performance rather than in ratings. Rated distinctiveness of faces correlated positively with hit rates to these items and negatively to rates of false positives (FPs) when the faces served as distractors, but there was a zero correlation between hit rates and FPs to the same items.

Bruce et al. (1994) used pictures of faces devoid of hair. In this paper, we first report our replication of the finding of two orthogonal components of typicality, obtained by using more natural images of faces shown with hair. We then go on to examine the possibility that PCA decomposition of facial images captures the important dimensions on which faces vary. We relate this decomposition to human performance on the same images. Our particular aim was to establish whether the two independent components of typicality (memorability and familiarity) can be accounted for independently by PCA decomposition. In the course of this exploration, we shall examine whether separate components of a face reflecting its shape and its surface texture give rise to different effects in the two components of typicality.

\section{EXPERIMENT 1}

\section{Method}

The aim of this experiment was to gather distinctiveness and memorability ratings on a set of faces to be used for the subsequent image analysis with PCA.

Materials. One hundred seventy-four black-and-white photographs of young adult males, normalized for interocular distance and eye position, were selected from the Aberdeen Frame Face Database (Shepherd, 1986). The people photographed had no facial hair or spectacles, had a neut ral expression, and their clothing was concealed by a dark gown tied at the neck. Photographic subjects were looking directly at the camera, in diffuse lighting.

Subjects. Thirty-four volunteer students, male and female, were paid to take part in the experiment.

Design. The image set was divided, at random, into two sets of 87 images, Set A and Set B. Subjects were asked to rate the faces in one of these subsets for distinctiveness by answering the question: "How easy would it be to spot this person at a train station?" The rating set was preceded by a familiarization set of nine faces drawn from the same population but not used in further analysis. This served three purposes: to orientate subjects on the type of faces and likely range of distinctiveness to be used; to acquaint them with the methodology (clicking a response box with a mouse pointer); and to reduce primacy effects in the following recall stage. Responses were made on a scale of 1 to 10 . Subjects were allowed to study each face for as long as they wished. Presentation order was randomized independently for each subject. This task has been used to collect ratings of distinctiveness in several previous studies (e.g., Bruce et al., 1994; Valentine \& Bruce, 1986a, 1986b).

Following this rating stage, subjects were asked to take part in a separate experiment (on object recognition) lasting about 10 minutes. They were then unexpectedly presented with the complete set of 174 faces, in sequence, and asked for each face, "Did you see this person before?" Subjects responded on a 10-point scale: $1=$ certain I did not see the face before, $10=$ certain I did see the face before. Once again, presentation of test faces was randomized independently for each subject.

This technique allows a number of direct measures to be taken for each face. First, it allows a mean distinctiveness rating, derived from the subjects rating faces in a particular set. Second, it allows a measure of hits corresponding to the certainty score of subjects who actually saw the face in the learning phase. Third, it allows a measure of false positives corresponding to the certainty score of subjects who did not see the face in the learning phase.

\section{Results}

Table 1 shows the mean ratings for subjects exposed to each half of the set of faces. The two sets show very similar levels of distinctiveness, hits and false positive ratings. Table 1 also shows estimates of $d^{\prime}$ and criterion. These estimates are calculated by taking hit and false positive scores for each face, counting responses of 6 and above as positives and responses of 5 and below as negative. Table 1 shows mean $d^{\prime}$ for faces that appeared in each of the two sets. Because we are averaging the results of single observations from each subject, the $d^{\prime}$ values are likely to be underestimated. However, the average $d^{\prime}$ value of 1.37 is remarkably similar to the value of 1.36 reported by O'Toole et al. (1994), who used the same calculation for a very different set of faces. Table 2 shows the correlation between the average subject responses for each face, broken down by subset, and jointly. These correlations once again demonstrate that whereas distinctiveness is correlated both with hit and distractor ratings, hit and distractor ratings are themselves uncorrelated. Figure 3 shows scatterplots of the data. This replicates a study using a different set of faces with hair concealed (Bruce et al., 1994), and again provides further support for the dissociation first described by Vokey and Read (1992). We have since replicated this pattern of data in two further studies in our laboratory, in one of which we used much smaller memory sets ( 16 tar- 
Table 1

Mean Scores for the Two Sets of Faces

\begin{tabular}{lccccc}
\hline & Distinctiveness & Hit Score & False Positive Score & $d^{\prime}$ \\
\hline Set A & 5.67 & 6.95 & 3.58 & 1.48 \\
Set B & 5.8 & 6.96 & 3.79 & 1.25 \\
\hline
\end{tabular}

gets and 16 distractors), so it does not seem to arise as a consequence of the large memory load. Furthermore, as we describe below, the dissociation does not appear to arise from order effects.

\section{Further Analysis}

In later sections of this paper, the images used in Experiment 1 will be analyzed with PCA. The intention is to establish whether PCA provides any account of the psychological dimensions revealed here. However, before describing these image analyses, we briefly report some further analyses of the data from this experiment.

Dimensions of subject performance. The striking finding in the data presented above is that hit and false positive scores dissociate. This means that faces that are easy to remember if subjects have seen them are not necessarily the faces that are easy to reject if subjects have not seen them. This finding has been treated in different ways by researchers in the past. Bruce et al. (1994) tried to account directly for the dimensions corresponding to hit and false positive scores observed in human data. In contrast, Vokey and Read (1992) used factor analysis on their subjects' ratings of typicality, memorability, attractiveness, familiarity, and likeability (this is the standard use of PCA for data reduction, not for face images) to derive two orthogonal components that they labeled "memorability" and "context-free familiarity."

In the analyses presented below, we have examined both direct and derived measures of human performance. In order to explore the structure of the data presented above, we performed factor analysis on the rating and performance data from these studies (note the contrast with the purely rating data of Vokey and Read, and of O'Toole et al., 1994). From the three scores available from each face (distinctiveness rating, hit scores, and false positive scores), we extracted two orthogonal factors. Table 3 shows the correlations between these factors and the subjects' rating and performance scores.

The first factor is heavily loaded onto distinctiveness, and also onto $d^{\prime}$, thereby justifying its label of memorability. As in the study by Vokey and Read (1992), we appear to have extracted a component that codes the abil- ity of subjects to recall having seen a face. The second factor is uncorrelated with both distinctiveness and $d^{\prime}$, but is heavily loaded onto criterion-the subjects' response bias for each face. This appears to capture the dimension labeled context-free familiarity by Vokey and Read; that is, it seems to capture the tendency for subjects to claim that they have seen the face before, irrespective of whether or not it appeared in the learning set.

Because there is no very good reason to claim that either the direct subject data (distinctiveness, hits, false positives) or the derived data (memorability and contextfree familiarity) should act as the standards against which subsequent performance of image analysis should be measured, we will examine the relation between PCA on images and both of these types of measure in later sections.

Between-subjects variability. Before attempting to relate subjects' data to the properties of the facial images, we need to examine the consistency of subjects. There are two reasons why this is necessary. First, we need to know whether there is general agreement about whether a face is distinctive, and whether the same faces are rejected or remembered. A general agreement would indicate (at least the possibility) that the measured dimensions have something to do with the faces themselves, rather than with idiosyncratic subject variables. Second, we need a measure against which to examine our subsequent analysis. Any analysis derived by synthetic means can be expected to correspond to subject data only to the extent that there is agreement among subjects.

In order to examine the consistency of subjects, we carried out further analysis of the data from distinctiveness, hit, and distractor scores. There are two plausible ways to examine consistency. The first two rows in Table 4 show the mean correlations between every pair of subjects within each group (each mean being composed of 136 correlation coefficients). The second two rows in Table 4 show a measure of consistency of subtotals of subject performance. Each group was split into two subsets (of 8 and 9 subjects), and the mean scores for each subset were correlated. This procedure was repeated 12 times with different random divisions into subsets. The second two rows in Table 4 show the mean correlations derived from this measure.

Table 4 shows an initially surprising result. The subjects appear to have been relatively consistent when assigning distinctiveness scores. On the other hand, they were much less consistent in terms of which faces they remembered from the learning phase. The measures in

Table 2

Correlations Between Subject Responses

\begin{tabular}{lcccc}
\hline & $\begin{array}{c}\text { Distinctiveness- } \\
\text { Hit }\end{array}$ & $\begin{array}{c}\text { Distinctiveness- } \\
\text { False Positive }\end{array}$ & $\begin{array}{c}\text { False Positive- } \\
\text { Hit }\end{array}$ & Distinctiveness- $d^{\prime}$ \\
\hline Set A & .55 & -.39 & -.17 & .51 \\
Set B & .4 & -.42 & .06 & .5 \\
Both & .49 & -.4 & -.08 & .5 \\
\hline Note-Critical value $r=.21$ for Sets A and B, .15 for combined set.
\end{tabular}



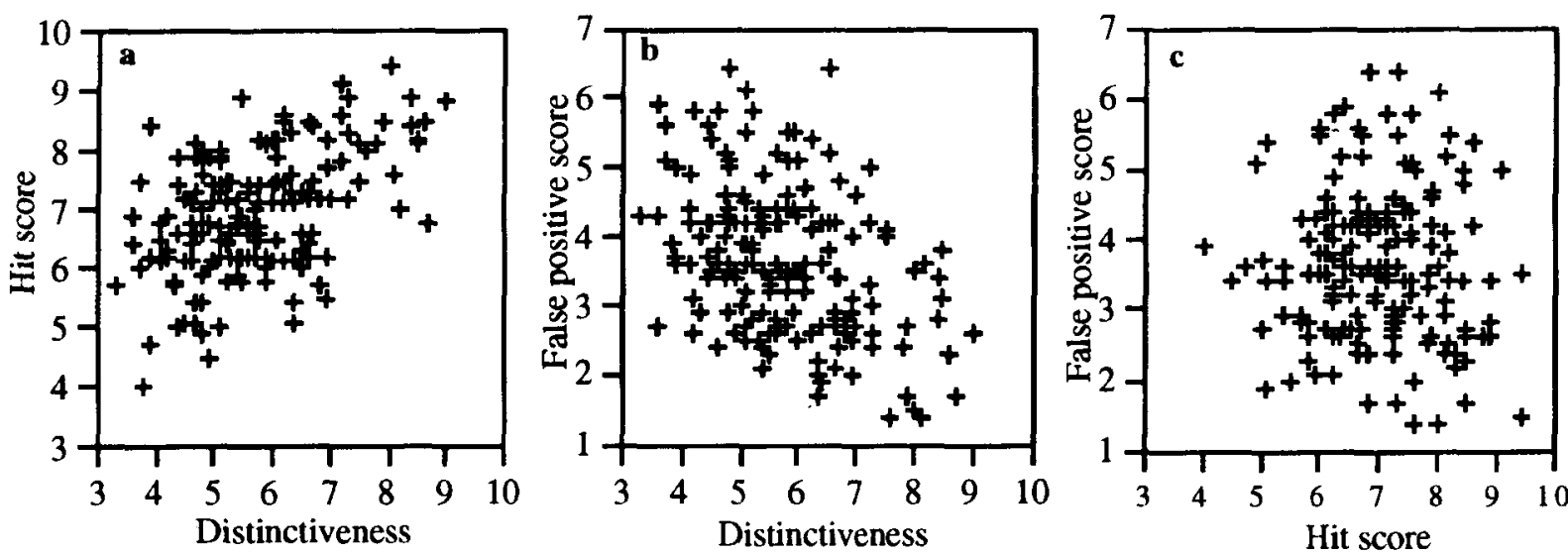

Figure 3. Scatterplots for all 174 faces showing (a) positive correlation between distinctiveness and hit score, (b) negative correlation between distinctiveness and false positive score, and (c) lack of correlation between hit and false positive scores.

Table 4 show a consistent pattern. The measure of distinctiveness has the highest consistency between subjects, followed by the false positive score, with hits showing the lowest consistency across subjects.

Despite this generally stable pattern of consistency, the overall levels are disappointingly low. The demands of this task were quite high on subjects; recall that subjects first rate a set of 87 faces, and subsequently have to rate a superset of 174 faces for whether they have been seen before. It is possible that the very large numbers of faces seen may have given rise to inconsistent behavior. Since the order of presentation was randomized independently for each subject, any effect of tiring would affect faces inconsistently.

We considered two artifactual reasons why subjects should fail to agree on hits. The first was the time spent studying each face during the rating phase. It seems plausible that faces looked at longer for some reason would be recalled better. However, analysis showed only 1 subject for which there was a significant correlation between observation time and hit score. Viewing time can therefore be eliminated as an explanation.

The second possible reason for the low consistency of hit scores is the randomized order of presentation. Although the initial display of nine faces in the familiarization phase should reduce possible primacy effects, it still seems possible that recency, or residual primacy effects, might have affected recall. A plot of order of presentation during the rating phase against hit score showed no such list-end effects, and we concluded that this was not a significant source of intersubject difference. However, a plot of hit score against presentation order during recall showed a significant negative correlation $(r=-.38, p<.05)$, as did a plot of false positive scores $(r=-.32, p<.05)$. A possible explanation for this is that subjects were aware that half the test faces had been presented for rating. Because many of the faces were quite similar, subjects might have responded positively at the start of the test, but increasingly negatively as their subjective ratio of hits became depleted. Whatever the reason, the tendency would contribute to the observed lack of correlation between subjects. We therefore replicated Experiment 1, but used fewer items and consistent ordering.

\section{EXPERIMENT 2}

\section{Method}

A subset of 80 of the previous faces were used: the top 10 and the bottom 10 faces rated for distinctiveness from Experiment 1 , and 15 each from the four intermediate ranges $(4-5,5-6,6-7$, $7-8)$. The ends of the range are thus overrepresented, relative to the population for Experiment 1. Twelve volunteer student subjects were recruited. None had taken part in Experiment 1. The procedure was exactly as in Experiment 1, except that the faces were presented in the same order on each occasion.

\section{Results}

As with Experiment 1, we present the data on consistency of subjects in two ways. Table 5 shows the mean correlations for each pair of subjects ( 15 pairs for each set). Table 5 also shows the grouped averages: Each group of 6 was split in half in each of the 10 possible ways to give average ratings values for each of the scores. As expected, the pairwise results were higher than those in

Table 3

Correlations Between Two Orthogonal Factors and the Human Data

\begin{tabular}{lccccc}
\hline \multicolumn{1}{c}{ Factor } & Distinctiveness & Hit & False Positive & $d^{\prime}$ & Criterion \\
\hline Memorability & .93 & .66 & -.6 & .74 & -.06 \\
Familiarity & .08 & .60 & .75 & -.16 & -.9 \\
\hline
\end{tabular}

Note-Critical value $r=.15$. 
Table 4

Mean Intersubject Correlations, Experiment 1

\begin{tabular}{cccc}
\hline Set & Distinctiveness & Hit & False Positive \\
\hline & \multicolumn{2}{c}{ Pairwise Correlations } \\
A & .28 & .09 & .13 \\
B & .23 & .04 & .17 \\
& \multicolumn{2}{c}{ Subset Correlations } \\
A & .79 & .45 & .63 \\
B & .71 & .28 & .56 \\
\hline
\end{tabular}

Note-Subset correlations were produced by random split.

Table 4. Removing the order effects and reducing the set size increased subject consistency. The grouped results are lower than those of Table 4 because of the smaller group sizes. However, the pattern of results is exactly the same as in Experiment 1: Subjects were most consistent in their distinctiveness ratings, followed by false positive scores, and least consistent on the hit rates. The same pattern of correlation is observed between the three sets of data for each face: a significantly positive correlation between distinctiveness and hit score $(r=.27, p<.05)$, a significantly negative correlation between distinctiveness and false positive $(r=-.36, p<.05)$, and a nonsignificant correlation between hit and false positive scores $(r=-.21, p>.05)$.

\section{DISCUSSION \\ Experiments 1 and 2}

In these experiments, we showed that hit rate and false positive scores were uncorrelated in subject data. This puzzling effect seems well grounded, as it has been demonstrated in ratings data as well as performance data, and we have replicated our performance data in several different studies, including one with only 16 faces in the rating set. In the next section we attempt to account for this effect.

In Experiments 1 and 2, we also showed that there was a robust pattern in the consistency of subjects. Subjects were in relatively high agreement about which faces were distinctive, behaved less consistently in false positives, and were least consistent in their hits. This leads us to make the following tentative proposal. It is possible that subjects make distinctiveness ratings on the basis of two dimensions, and that these are reflected in the hit and

Table 5 Mean Intersubject Correlations, Experiment 2

\begin{tabular}{|c|c|c|c|}
\hline Set & Distinctiveness & Hit & False Positive \\
\hline \multicolumn{4}{|c|}{ Pairwise Correlations } \\
\hline A & .35 & .25 & .30 \\
\hline B & .42 & .14 & .21 \\
\hline \multicolumn{4}{|c|}{ Subset Correlations } \\
\hline A & .68 & .44 & .59 \\
\hline $\mathrm{B}$ & .68 & .32 & .44 \\
\hline
\end{tabular}

false positive scores. The larger consistency of false positive scores may reflect a general property of these faces-perhaps their similarity to the population of faces as a whole. The hit score, on the other hand, may reflect idiosyncratic knowledge of faces by subjects. It is possible that certain subjects remember particular faces because they are similar to someone known to the subject. This may explain the inconsistency of these ratings.

This suggestion is clearly speculative, and we shall return to a discussion of these issues at the end of the paper. We now turn to an analysis of the images used in these experiments.

\section{ANALYSIS OF IMAGES}

In the sections above, we have presented data from subjects who were shown sets of facial images. In this section we shall present analyses of these images themselves. The aim is to establish whether characteristics of these images can be found that might account for patterns in the human data. As described in the introduction, we are particularly interested in the possibility that PCA of facial images can reveal aspects of faces that predict psychological effects. This issue has been addressed by O'Toole et al. (1994) in respect to the otherrace effect. These researchers found that the advantage for recognition of faces from one's own race can be captured in PCA analysis of images, assuming a larger exposure to one particular race. In the present paper, we aim to examine the relation between PCA and the psychological data on distinctiveness. In particular, we aim to explore the possibility that PCA can account for the separate effects of hits and false positives, as described above. In addition to extending the range of psychological data addressed by PCA, we shall also examine the possibility that the separate analysis of shape and texture may inform this analysis.

The aim of PCA is typically to reduce the dimensional size of the input set. Given the procedure of ordering components, most of the variance is captured by the early factors. In the studies that follow, we shall usually consider only the first 20 eigenfaces, as our observations are that the first 20 dimensions are sufficient to capture most of the variance in the input set. However, we shall analyze this more explicitly in a later section.

Having performed PCA on images, we will use two types of information to relate the images to psychological data. First, we can analyze the PCA outputs for a particular face: What are the weights allocated to it for each derived eigenface? We refer to this set of values as the face's spectrum. Second, we can ask how good the coding is of a particular face. If, for example, we were to reconstruct a face from its spectrum of (say) the first 20 outputs, how well would the face be reconstructed? We refer to the measure derived in this way as the reconstruction error of a face.

In order to conceptualize this more clearly, consider the psychological dimension of distinctiveness. PCA 
captures a particular notion of distinctiveness: that of being a long way from the mean value. Specifically, it identifies the axes with maximal variance. If, in capturing variations from the mean in either the image gray levels or the measured shape of the face, PCA captures something of what our subjects regard as distinctive, then faces rated as distinctive should tend to have large component values. In other words, we might expect that distinctive faces are allocated values that are distant from the mean on some of the components. If we now consider reconstruction errors, we might predict that distinctive faces are coded less well by the early eigenfaces, precisely because the early components capture variations common to many faces, which distinctive faces, by definition, do not share so much. Distinctive faces should therefore have high reconstruction errors. We shall investigate both these possibilities in the following sections, as well as consider the relation between PCA and the other psychologically derived measures.

Finally, there is an added complication in considering PCA of images. The above discussion assumes that the same set of images is used to generate PC coefficients and for subsequent testing. However, it is equally plausible to generate principal components with one set of images and to code a new set on the components generated. In this case, we refer to the outputs as the reflection of the second set through the components of the first. This procedure may be seen as more similar to the human experiment, in which experience derived from previously seen faces is brought to bear on a novel set. It can be used to generate data for either the spectrum or reconstruction error analyses described above, and in the following we shall examine both same-set and reflection data.

PCA offers a possible explanation of the dissociation between well-remembered and well-rejected faces. It might be expected that faces that are badly coded by the system (human or computer) would be poorly remembered. Such a face would be regarded as distinctive, in the sense of having a high reconstruction error. It should be easy to reject such a face, on the grounds that, by definition, it is outside the range of familiar faces. These, then, would be the faces that are distinctive and well rejected but poorly remembered. Conversely, a high PC spectrum output from a face that is distinctive, but within the space coded by the coefficients, might correlate with those that are better remembered due to particular similarity to individual faces known by the subject. Unfortunately, this is difficult to test experimentally, because the population of known faces will differ among subjects. For a distinctive face in the test set to be wellcoded by the PCs would require another similar distinctive face to be present in the generation set, which, given the modest size of the sets, seems unlikely.

\section{Materials: Image Processing}

For computer processing, the $256 \times 256$ images shown to the subjects were reduced to $64 \times 64$ with 256 levels of gray. In the following analyses, PCA is performed on both untransformed and shape-free images. To generate the shape-free images, a shape map for each face was defined manually, specifying the $x$-, $y$-coordinates of 35 locations of features, such as eyes and nose, and the periphery of chin and hair (Figure 1). These coordinates were used to produce shape-free face images by "morphing" to the average shape using bilinear interpolation. The background of both shaped and shape-free images was removed by setting the pixels outside of the area bounded by the shape map to zero.

\section{Multiple Regression}

We first attempt to account for the human data using multiple linear regression. The approach adopted is to use PC outputs (i.e., spectrum values) for each face to predict the human data. Taking distinctiveness as an example, we might expect there to be large correlations (multiple $R$ ) between the absolute spectrum values and distinctiveness. This is because, as explained in the previous section, we might predict that faces rated as distinctive will have large (discrepant) values on some or all of the components.

Intuitively, large absolute values of $\mathrm{PC}$ outputs might contribute to a face's distinctiveness, given that distinctiveness may lie on both sides of the mean. However, in this study we were also attempting to predict other human data. We used PC outputs to predict the data collected on hit and false positive scores and also to predict the derived dimensions labeled memorability and contextfree familiarity (see above). In contrast to distinctiveness, there is no simple intuitive relation between these scores and PC outputs. We therefore used both absolute and raw (signed) PC outputs as predictor variables in the following study.

Whole face set. In the first multiple regression study, we used the first 20 components derived from a PCA of the entire set of 174 images. The somewhat arbitrary decision to use 20 components was based on the observation from initial tests that there was little sign of consistent correlations between any higher components and the subject rating data. Both raw and absolute values were entered as predictor variables. Variables were entered by stepwise addition, with the criterion that to enter a variable must increase the multiple $R$ significantly $(F>3.84)$.

In order to study the relative contributions of shape and texture to these correlations, we repeated this procedure four times, using predictor variables from PCA of four different sources. These were as follows: (1) the original images, adjusted to bring their eyes to the same coordinates; (2) the shape-free images, morphed to fix the coordinates of 35 locations; (3) the shape vectorsthe set of $35(x, y)$ pairs for the morphing coordinates; and (4) the first 13 components from the shape-free images and the first 7 from the shape vectors.

Data Set 1 gives a measure of this technique for untransformed facial images, and Data Sets 2 and 3 analyze face texture and face shape separately. Data Set 4 represents an attempt to recombine shape and texture after separate PCA on these two sources. If these two aspects of a face are analyzed separately, each must undergo the 
PCA before being brought together. This ratio of texture to shape components (approximately 2:1) was chosen on the basis of an inspection of the correlations given by individual component outputs, which were mostly low for shape components higher than 7 (see also Table 8). Note that the shape vector has only 70 entries per image. Extracting 20 components from this set accounts for much more of the total variance than 20 components of the 4,096-dimensional-image data. We used the same number of components to facilitate comparison between the resultant multiple- $R$ values.

The multiple- $R$ values between the component outputs and the human data for these four cases are shown in Table 6. With 40 variables, it is to be expected that some apparently significant correlations will arise by chance. An estimate of this chance level of multiple $R$ was obtained by randomizing the order of the human data and rerunning the multiple regression. This was repeated 100 times, giving an average multiple $R$ of .28 , which we take to be chance performance.

There are several points to note from this table. Distinctiveness is predicted at reasonably high levels by all the different types of data. As might be expected, removing the shape from the images reduced the level of prediction achieved (multiple $R=.51$ vs. .40 ), and the shape-alone and shape-free images achieved roughly equivalent levels of prediction (.40 vs. .42). Hit scores were predicted poorly (at chance) by the shape-free and shape-alone data, and better by the full (untransformed) data, and by the combination of shape and shape-free data. Perhaps the most surprising results come from predictions of false positives. It seems that removing the shape from a face makes a substantial improvement in the correlation with false positive scores (multiple $R=$ .36 vs. $.50, F$ test, $p<.01$ ). Furthermore, the shape vector alone does not predict FP rates at above chance levels. Finally, the derived components memorability and familiarity behave similarly to distinctiveness and false positive, respectively. Using some components from both shape vector and the shape-free images appears to give the best of both, with correlations for all the subject data being near their best.

We are unable to claim too much from these results, due to the dangers inherent in multiple regression. These are highlighted by the results for hit score, one of which is considerably below the value expected by chance. Small variations in the data may make the difference between a variable entering the equation or not, with consequent effects on the reported multiple $R$. Although differences such as those between .51 for dis- tinctiveness and .33 for hit score with full faces or .40 for distinctiveness and shape free are formally significant ( $F$ test, $p<.01$ ), the evident noise makes such comparisons unconvincing. We therefore adopted the following method.

Random segmentation of the face set. The set of faces was split randomly in half. One half was used to generate PC coefficients. These coefficients were then used to analyze the other half. The outputs obtained were used to perform multiple regression on the corresponding human data. This process was repeated 100 times to obtain average multiple- $R$ values. It is not usually safe to average correlation coefficients because of their nonnormal distribution; furthermore, the samples are not independent, being drawn from the same complete set. However, we have no reason to suppose that the distribution of correlations for the various human data will be different. Averaging should therefore not affect the rank ordering of the correlations obtained, and we shall concentrate on this ranking below. Averaging serves to smooth out the effects of random correlations and produces a clearer pattern of results, shown in Table 7.

Estimates of the chance correlation were obtained as before, by randomizing the order of the human data and rerunning the multiple regression for each of the $100 \mathrm{seg}$ mentations of the data set. They came out very consistently, with none differing significantly from an overall average of .336 . This number is larger than the value of .28 obtained above, because we were using only half the faces on each test. For these randomized controls, an analysis of variance comparing 100 runs from each of four preprocessing methods over five subject variables showed no effect of variable (distinctiveness, hit, etc.) $[F(4,396)<1]$, no effect of preprocessing (shape, shapefree, etc.) $[F(3,297)<1]$, and no interaction $[F(12,1188)=$ $1.24, p>.2]$. We therefore assume that the expected chance correlations are the same for all the conditions shown in Table 7 and that the results may therefore be compared directly with each other. All of the multiple- $R$ values shown are significantly above the random value of .336 ( $t$-test, $p<.01)$ except those from the shape vector to false positive score and context-free familiarity. Differences between the multiple- $R$ values were tested using the Mann-Whitney $U$ test on the 100 samples for each condition, at $p<.01$. To give a feel for the consistency of the results, and noting the problems of averaging correlation coefficients, the standard errors on these data are all approximately .01 .

In summary, we (1) split the face set in half at random, (2) extracted principal components from one half, (3) re-

Table 6

Multiple Regression Values for PC Outputs (Spectrum) Predicting Human Data, Using Whole Set of Faces

\begin{tabular}{lccccc}
\hline & Distinctiveness & Hit & False Positive & Memorability & Familiarity \\
\hline 1. Full & .51 & .33 & .36 & .51 & .34 \\
2. Shape-free & .40 & .28 & .50 & .40 & .40 \\
3. Shape vector & .42 & .19 & .25 & .44 & .22 \\
4. Shape-free + shape & .49 & .36 & .44 & .43 & .40 \\
\hline
\end{tabular}


Table 7

Averaged Multiple Regression Values for PC Outputs (Spectrum) Predicting Human Data

\begin{tabular}{lccccc}
\hline & Distinctiveness & Hit & False Positive & Memorability & Familiarity \\
\hline 1. Full & .51 & .42 & .42 & .48 & .38 \\
2. Shape-free & .48 & .40 & .49 & .44 & .44 \\
3. Shape vector & .48 & .37 & .30 & .42 & .32 \\
4. Shape-free + shape & .52 & .43 & .48 & .49 & .42 \\
\hline
\end{tabular}

Note-Data averaged over 100 random half splits of the face set

flected the remaining faces through these components, (4) performed multiple regression between the raw and absolute values of the first 20 component outputs (13 image and 7 shape for the combined type) with each of the five subject measurements, (5) randomized the order of the subject measurements and repeated the multiple regression to obtain an estimate of the chance correlations, and (6) repeated Steps $1-5$ one hundred times for each of the four preprocessing types and averaged the results within preprocessing type and subject measurement pairs.

The pattern of results confirms that suggested by Table 6:

1. With the full faces, the results for hit and false positive scores were the same, with that for distinctiveness significantly higher.

2. Moving to shape-free faces caused a significant drop for memorability, a small downward trend for distinctiveness and hit score, but a significant increase for false positive score and familiarity.

3 . The values for shape vector alone were significantly worse than for full-face for all variables. False positive and familiarity were at the random values. Hit score was above chance, but just barely.

4. Adding the shape vector PCs to those from shapefree images did best of all. The multiple $R$ for distinctiveness was significantly better than that from the shapefree images and was indistinguishable from that from the shaped images, whereas that for false positive was significantly better than the result from shaped faces, and was indistinguishable from the shape-free performance. The three values for hit did not differ significantly.

As far as we are aware, this is the first demonstration of the possible psychological relevance of shape averaging. The process increases the ability of PCA to extract information that leads people to say "yes" when they have not seen a face before, which affects both false positive and familiarity scores. Conversely, there appears to be no information available from the shape vector about false positives. As far as we can account for human false positive scores, the information comes from fine detail in the image, which we refer to as the texture. We shall return to general discussion of these issues at the end of the paper. However, we now examine the contribution of individual components to the psychological predictions described here.

\section{Individual Component Correlations}

The multiple regression results suggest that different aspects of the images carry information about memorability and familiarity. Further insight into the nature of the features underlying these two dimensions might be obtained by examining the loading of the individual principal components. In this section, we examine whether particular components carry information specific to particular psychological dimensions.

There are a number of ways that this might be done. The first is to generate components from the complete set of faces, echoing the first procedure for multiple regression above. We may then look at the individual correlations between each component and the subject ratings for each face. Because this can be done only once, there are uncertainties about the replicability of the results.

A second approach is to split the face set randomly as before and produce average correlations for each component. In addition to the usual problems of averaging correlation coefficients, there is an additional problem of potential inconsistencies in the order of principal components. With the set split in half, we have 87 data points in the 4,096-dimensional-pixel space: clearly a very sparse sampling. Given the relative homogeneity of our images, this may not be too problematic, but we can nonetheless expect the derived components to vary between runs. For instance, the information contained in Component 7 on one occasion might appear as Component 8 in another, or be redistributed among two or more different components. Visual inspection of the eigenfaces from different runs suggests that this starts happening as early as Component 3 or 4 . Any correlations between individual components and the subject ratings will therefore be unstable. Averaging the results will lead to correlations being spread over a number of neighboring components.

This problem of uncertain distribution of variance may also affect the first method (using all the images to generate components). It could be that there is useful information about distinctiveness that usually occurs, say, around Component 10 , but that on this occasion is distributed among several other components. These will each show a small, apparently nonsignificant correlation, where a slight change would lead to a significant correlation for Component 10. Averaging would allow such effects to show through. 
A third approach is to look at the components actually used in the multiple regression equation. This has the advantage of identifying whether the various components are accounting for different aspects of the subject ratings. The first four components might all correlate with distinctiveness, but all be capturing the same part of the variance. The multiple regression equation should include only the most significant of these, giving a clearer indication of the most important components. As before, we may look either at the regression equation for the whole set of faces, with consequent doubts about repeatability, or use the random sampling technique. The same problem about inconsistent components will apply: Over a number of runs we would expect to find neighboring clusters of components, at most one or two of which appear in any particular regression equation.

Table 8 shows the usage of components in the 100 multiple regression equations generated above. Almost all the components occurred at least once, as would be expected because of chance correlations. During the 100 control runs with randomized subject ratings, each component occurred on average 4.67 times. The binomial distribution then requires more than 12 occurrences in 100 trials for $p<.001$ (such a low $p$ value being used because we have 40 variables). Table 8 reports the components that occurred more than 12 times.

The pattern of component usage confirms the multiple regression results, with false positive and familiarity showing an increase in components used when going from full image to shape free, while the other three showed a decrease. The relative usage of information from the shape vector and the image is shown clearly by the combined component results, with false positive and familiarity loading heavily onto the image components, and the other three onto shape components. Although our familiarity measure is derived about equally from the hit and false positive scores (Table 3 ), it behaves more like the latter. Only the use of Components 4 and 6 from the shape vector echoes the loading of hit score.

Within each preprocessing category, there is little in common between false positive and the other two direct subject ratings. Thus false positive uses the first two components from the full image set, whereas distinctiveness and hit use several in the range $3-12$. A striking result not indicated by Table 8 is the frequency with which false positive used the first component: 73 of the 100 runs for the full images and 82 for the shape-free im- ages. The other "frequent" occurrences are typically in the range $20-40$. One reason for this may simply be that it is the first component, and therefore relatively stable. As indicated above, it may be that there is a similar amount of information about distinctiveness that occurs somewhere in the range 7-10 for the full images, inconsistently because of the variability on the principal components. Further analysis of the data supports this suggestion, showing that at least one of these components occurs in 78 of the 100 equations for distinctiveness and that never more than two of them occur together.

\section{Reconstruction Errors}

We now turn to another measure of PCA performance: reconstruction error. This gives us a measure of how well a particular face is coded, when included in the whole data set. The purpose of this analysis is to explore the possibility that distinctive faces are coded less well by PCA than are typical faces.

O'Toole et al. (1994) reported reconstruction error using a normalized cosine error, where 1 means perfect reconstruction and smaller numbers are worse. A possible alternative is simple Euclidean distance between the input and reconstructed images (i.e., the length of the vector between the two points in 4,096-dimensional-image space representing the face and its reconstruction). If the image vectors are normalized to unit length before the distance is computed, this measure differs from O'Toole's only in the cosine nonlinearity. Tests showed much larger correlations if the vectors were not normalized.

Although our stated aim is to examine whether distinctiveness can be captured as a correlate of "goodness of coding" in PCA, the goodness of coding is itself dependent on a number of parameters. In particular, the more components that are extracted from the set, the better the general coding. In this study we calculated the reconstruction error for different numbers of extracted components. These measures were then correlated with the psychological data.

Figure 4 shows the correlation between the unnormalized Euclidean reconstruction error and each of the three direct and two derived subject data sets as the number of components used for the reconstruction is varied. The complete set of 174 full (i.e., not shape-free) images was used both to generate the components and to test reconstruction. Note that we are considering far more than the 20 components used for the multiple regression studies: Although higher components showed little direct corre-

Table 8 Usage of Components in Multiple Regression Equations, From Same 100 Runs as Those in Table 7

\begin{tabular}{|c|c|c|c|c|c|}
\hline Type & Distinctiveness & Hit & False Positive & Memorability & Familiarity \\
\hline Full & $3,4,7,8,9,10,12, \mathrm{~A} 1, \mathrm{~A} 2$ & $4,6,9,10, \mathrm{~A} 1, \mathrm{~A} 2$ & 1,2 & $3,4,8,9,10,11,19, \mathrm{~A} 1, \mathrm{~A} 2$ & 1 \\
\hline Shape-free & $2,3, \mathrm{~A} 1, \mathrm{~A} 15, \mathrm{~A} 17$ & $2,7,17, \mathrm{~A} 2$ & $1,2,6,7$, A5 & $2, \mathrm{~A} 1, \mathrm{~A} 15$ & $1,6,7,19$, A5 \\
\hline Shape vector & $4,6,19, \mathrm{~A} 1, \mathrm{~A} 7, \mathrm{~A} 9, \mathrm{~A} 18$ & $2,4,6, \mathrm{~A} 7$ & A 7 & $4,6, \mathrm{~A} 1, \mathrm{~A} 7, \mathrm{~A} 18$ & 4,6 \\
\hline Combined: Shape & $1,4,6, \mathrm{~A} 1, \mathrm{~A} 6, \mathrm{~A} 7$ & $4,7, A, A 7$ & A7 & $1,4,6, \mathrm{~A} 1, \mathrm{~A} 7$ & \\
\hline Image & $2, \mathrm{Al}$ & 2 & $1,2,6,7$, AS & $2, \mathrm{Al}$ & 1,6 \\
\hline
\end{tabular}

Image Note-Components listed occurred more often than expected by binomial distribution $(p<001)$. Al refers to absolute value of Component 1 , and so forth. 
lation with the subject data, they all had a (gradually diminishing) effect on the reconstruction error.

To understand Figure 4, consider the first (leftmost) points. If only a small number of components is used to reconstruct a face, then the correlation between reconstruction error and distinctiveness is high. This means that faces rated as highly distinctive are reconstructed badly. However, the correlation between reconstruction error and false positive score is negative: That is, faces that are easy to reject, and so have a low false positive score, are reconstructed badly. Typical faces, which are poorly remembered and rejected, are relatively well coded, with low error.

The striking result shown in these graphs is the reversal of the sign of most of the correlations as the number of components is increased. This is most easily understood by considering distinctiveness. Each additional component will accommodate as much of the remaining variance as possible. Initially this is best done by coding features common to many images. Because lack of shared features is one definition of distinctiveness, these early components code average faces better than unusual ones. Distinctive faces therefore have a high reconstruction error. As the number of components is increased, there comes a point when there is little variance common to several images left to be accommodated. Now the best strategy for reducing variance is to cover those distinctive faces that were poorly coded by the early components. The correlations reverse, so that above about 50 components, distinctive faces are better coded than those that are more average. If the analysis were continued beyond 100 components toward the number of face images used, the correlation would fall back to zero along with the reconstruction error.

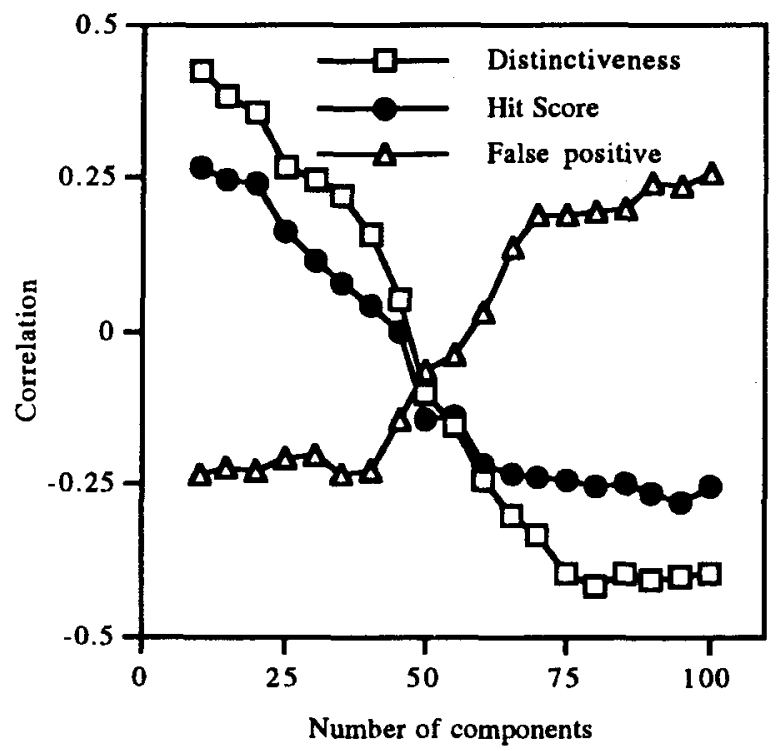

Distinctiveness and (derived) memorability behave almost identically, with hit score qualitatively similar, but at lower correlations. False positive score behaves almost like a mirror image, but crosses zero at closer to 60 components. The derived factor familiarity here behaves as a combination of hit and false positive. Correlation is close to zero, with only a mild negative excursion in the middle range, where hit score has dropped and false positive is still negative.

\section{GENERAL DISCUSSION}

The studies described above were performed to analyze the relationship between human face processing and structural properties of face images. As in previous work in this field (Abdi et al., 1995; O'Toole, Abdi, et al., 1991; O'Toole, Deffenbacher, et al., 1994), we have attempted to capture psychological effects in terms of the statistical properties of images, as revealed by PCA. We now summarize the data and offer some conclusions.

The psychological data on hits and false positives do not correlate. This means that faces that are easy to recognize as having been seen are not necessarily those that are easy to reject when they have not been seen. Some of the data described above suggest that we may be able to separate properties of the face that give rise to these two dimensions.

The studies of reconstruction error show that when one uses only the early components, faces that are badly coded have a high hit rate, whereas faces that are well coded have a high false positive rate. In other words, faces that are discrepant on these components are easy to recognize as having been seen, whereas faces that are not discrepant on these dimensions are those most likely to

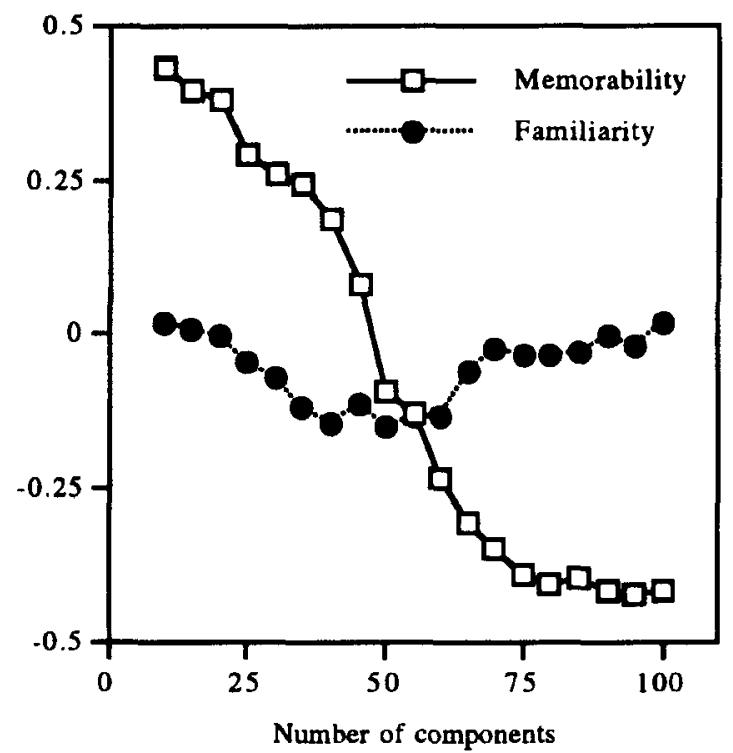

Figure 4. Correlation between the reconstruction error and the subject ratings for each face as the number of components used for the reconstruction is varied. 
be falsely identified as having been seen. When one uses a large number of components, this pattern reverses. This seems to indicate that it is the early components that give rise to false positives, whereas later components give rise to hit rate. This tentative conclusion is supported by the studies examining the individual components (Table 8). These show that, for full face images, it is the very early components that are most commonly used to predict false positives, whereas the components that predict hits tend to be drawn from later in the spectrum.

These suggestions are consistent with an intuitive notion of what is captured by the early and late components derived from PCA. The early components code very general information, extracting information common to all faces in the set. In general, we might say that these components define the range of face-like patterns (of pixel intensities). However, later components begin to pick up individual variation, as is shown by the reversal of effects in Figure 4. It is to be expected that hit and false positive scores, which are uncorrelated, would therefore load onto different, inherently orthogonal principal components. It is intuitively sensible that it should be the false positive score that loads onto the earliest components.

Further support for this view can be found in the separate analysis of shape and shape-free faces. Data from the multiple regression studies show that the shape-free faces capture the false positive data best of all (Tables 6 and 7). In other words, it is variation in what we have called texture that gives rise to false positives; variation in shape seems to make no contribution to the chances that a face will be falsely recognized.

Once again, examination of the data from individual components seems to support this. Table 8 (fourth line) shows the contribution of different components to the data from combined shape and shape-free information. Components from the shape-only information appear to load specifically onto hit scores, whereas components from the image (shape-free) information load onto false positive scores.

It appears quite clear from the data presented here that false positives arise as a consequence of a face's similarity to the general population. Furthermore, the measure of similarity used in this account does not include information about face shape, but rather information about coloration or texture. The interpretation of data from hits (and correspondingly from distinctiveness overall) is less easy to explain. It appears that dimensions giving rise to hits do include some information about the shape of a face. This lends some support to the notion of norm-based coding, as discussed in the introduction. Deviations from an average shape appear to predict the accuracy with which subjects identify a face as having been seen. However, shape does not account for the whole effect. Table 7 shows that the best predictor of hits (and distinctiveness) arises from separate analysis of shape information and texture information, subsequently brought together.

In the discussion of Experiments 1 and 2, we suggested that hit scores may be determined partly by sub- jects' idiosyncratic knowledge of people. Perhaps subjects score hits partly because a particular face reminds them of an acquaintance. This is consistent with the low levels of subject agreement on hit scores, and with the general finding that the later (more detailed) components tend to load on hits. We are now conducting experiments to test this hypothesis further.

In conclusion, it appears that we have isolated some of the separate information that gives rise to psychological properties of face perception. In particular, we have concentrated on subjects' hit and false positive scores in remembering faces. We have not done this by breaking down images of faces into everyday components like noses, chins, and so forth. Rather, we have extracted statistical properties of the images. Furthermore, we have shown that a decomposition of these images into separate shape and texture information makes it easier to account for some of the psychological data, suggesting that this distinction may also be made by the human system.

Although this paper reports some success in relating human data to statistical properties of images, we have not provided evidence that the PCA performed here is the best way to capture these data. The PCA was performed directly on the image pixels, for simplicity and because this is the approach taken by other workers. However, it is clear that the human visual system performs various types of filtration relatively early in its processing. It is quite possible that a more complete account would rely on PCA of filtered images, and we have begun to investigate the effects of such preprocessing (Hancock, Burton, \& Bruce, 1995).

Finally, the analyses presented here may be taken to support the general notion that image-based statistics provide some insight into human face processing. This contrasts with accounts based on local distance measures or surface-based measures as described in the introduction. However, PCA is just one of a broad range of image-based statistical techniques available (see, e.g., Brunelli \& Poggio, 1993, and Wurtz, Vorbruggen, \& von der Malsburg, 1990, for alternative techniques). Further research is required in order to establish whether the results described here are tied to the particular PCA analysis chosen, or are a general consequence of any imagebased technique.

\section{REFERENCES}

Abdi, H., Valentin, D., Edelman, B., \& O'Toole, A. J. (1995). More about the difference between men and women: Evidence from linear neural network and principal component approach. Perception, 24, 539-562.

Bartlett, J. C., \& SearCY, J. (1993). Inversion and configuration of faces. Cognitive Psychology, 25, 281-316.

Benson, P. J., \& Perret, D. I. (1991). Perception and recognition of photographic quality facial caricatures: Implications for the recognition of natural images. European Journal of Cognitive Psychology, 3, 105-135.

BIEDERMAN, I. (1987). Recognition by components: A theory of human image understanding. Psychological Review, 94, 115-147.

Bruce, V., Burton, A. M., \& Dench, N. (1994). What's distinctive about a distinctive face? Quarterly Journal of Experimental Psychology, 47A, 119-141. 
Bruce, V., Burton, A. M., Dench, N., Hanna, E., Healey, P., Mason, O., Coombes, A., Fright, R., \& Linney, A. (1993). Sex discrimination: How do we tell the difference between male and female faces? Perception, 22, 131-152.

Bruce, V., Coombes, A., \& Richards, R. (1993). Describing the shapes of faces using surface primitives. Image \& Vision Computing, 11, 353-363.

Bruce, V., Hanna, E., Dench, N., Healy, P., \& Burton, A. M. (1992) The importance of "mass" in line drawings of faces. Applied Cognitive Psychology, 6, 619-628.

Bruce, V., Healy, P., Burton, M., Doyle, T., Coombes, A., \& LinNEY, A. (1991). Recognising facial surfaces. Perception, 20, 755-769.

Bruce, V., \& Young, A. (1986). Understanding face recognition. British Journal of Psychology, 77, 305-327.

Brunelli, R., \& PogGio, T. (1993). Face recognition-Features versus templates. IEEE Transactions on Pattern Analysis \& Machine Intelligence, 15, 1042-1052.

Burton, A. M. (1994). Learning new faces in an interactive activation and competition model. Visual Cognition, 1, 313-348.

Burton, A. M., Bruce, V., \& Dench, N. (1993). What's the difference between men and women? Evidence from facial measurement. Perception, 22, 153-176.

Burton, A. M., Bruce, V., \& Johnston, R. A. (1990). Understanding face recognition with an interactive activation model. British Journal of Psychology, 81, 361-380.

Craw, I., \& Cameron, P. (1991). Parameterising images for recognition and reconstruction. In P. Mowforth (Ed.), Proceedings of the British Machine Vision Conference. Berlin: Springer-Verlag.

Davies, G. M., Ellis, H. D., \& ShePherd, J. W. (1978). Face recognition accuracy as a function of mode of representation. Journal of $A p$ plied Psychology, 63, 180-187.

Ellis, H. D. (1986). Processes underlying face recognition. In $\mathrm{R}$. Bruyer (Ed.), The neuropsychology of face perception and facial expression. Hillsdale, NJ: Erlbaum.

Farah, M. J., O'Reilly, R. C., \& Vecera, S. P. (1993). Dissociated overt and covert recognition as an emergent property of a lesioned neural network. Psychological Review, 100, 571-588.

Hancock, P. J. B., Burton, A. M., \& Bruce, V. (1995). Preprocessing images of faces: Correlations with human perceptions of distinctiveness and familiarity. In Proceedings of the IEE Fifth International Conference on Image Processing and Its Application. London: IEE.

HAY, D. C., \& Young, A. W. (1982). The human face. In A. W. Ellis (Ed.), Normality and pathology in cognitive functions (pp. 173-202). London: Academic Press.

Hayes, T., Morrone, M. C., \& Burr, D. C. (1986). Recognition of positive and negative bandpass-filtered images. Perception, 15, 595-602.

KirBy, M., \& Sirovich, L. (1990). Applications of the KarhunenLoeve procedure for the characterisation of human faces. IEEE Transactions on Pattern Analysis \& Machine Intelligence, 12, 103-108.

NosoFsKY, R. M. (1986). Attention, similarity, and the identification- categorization relationship. Journal of Experimental Psychology: General, 115, 139-157.

O'Toole, A. J., Abdi, H., Deffenbacher, K. A., \& Bartlett, J. C. (1991). Simulating the "other-race effect" as a problem in perceptual learning. Connection Science: Journal of Neural Computing, Artificial Intelligence \& Cognitive Research, 3, 163-178.

O'Toole, A. J., Deffenbacher, K. A., Valentin, D., \& Abdi, H. (1994). Structural aspects of face recognition and the other race effect. Memory \& Cognition, 22, 208-224.

Rhodes, G. (1988). Looking at faces. First-order and second-order features as determinants of facial appearance. Perception, 17, 43-63.

Rhodes, G., Brake, S., \& AtKinson, A. P. (1993). What's lost in inverted faces? Cognition, 47, 25-57.

Rhodes, G., BRENNEN, S., \& CAREY, S. (1987). Identification and ratings of caricatures: Implications for mental representations of faces. Cognitive Psychology, 19, 473-497.

SHEPHERD, J. (1986). An interactive computer system for retrieving faces. In H. Ellis, M. Jeeves, F. Newcombe, \& A. Young (Eds.), Aspects of face processing (pp. 398-409). Dordrecht: Martinus Nijhoff.

Tanaka, J. W., \& Farah, M. (1993). Parts and wholes in face recognition. Quarterly Journal of Experimental Psychology, 46A, 225-245.

Turk, M., \& Pentland, A. (1991). Eigenfaces for recognition. Journal of Cognitive Neuroscience, 3, 71-86.

VALENTINE, T. (1991). A unified account of the effects of distinctiveness, inversion and race in face recognition. Quarterly Journal of Experimental Psychology, 43A, 161-204.

Valentine, T., \& Bruce, V. (1986a). The effects of distinctiveness in recognising and classifying faces. Perception, 15, 525-536.

Valentine, T., \& Bruce, V. (1986b). Recognising familiar faces: The role of distinctiveness and familiarity. Canadian Journal of Psychology, 40, 300-305.

VALENTINE, T., \& ENDO, M. (1992). Towards an exemplar model of face processing: The effects of race and distinctiveness. Quarterly Journal of Experimental Psychology, 44A, 671-703.

VoKey, J. R., \& READ, J. D. (1992). Familiarity, memorability, and the effect of typicality on the recognition of faces. Memory \& Cognition, 20, 291-302.

Wurtz, R. P., Vorbruggen, J. C., \& von der Malsburg, C. (1990). A transputer system for the recognition of human faces by labeled graph matching. In R. Eckmiller, G. Hartmann, \& G. Hauske (Eds.), Parallel processing in neural systems and computers (pp. 37-41). Amsterdam: Elsevier.

Young, A. W., \& BRuce, V. (1991). Perceptual categories and the computation of grandmother. European Journal of Cognitive Psychology, 3, 5-49.

Young, A. W., Hellawell, D., \& Hay, D. C. (1987). Configural information in face perception. Perception, 16, 747-759.

(Manuscript received December 7, 1994; revision accepted for publication June 16, 1995.) 